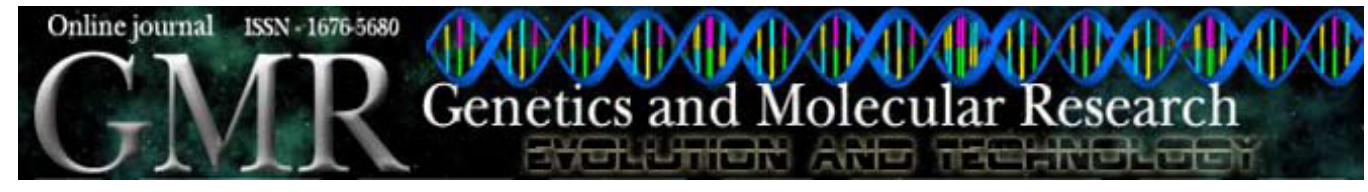

\title{
Understanding bamboo flowering based on large-scale analysis of expressed sequence tags
}

\author{
X.C. Lin ${ }^{1,3,5}$, T.Y. Chow ${ }^{2}$, H.H. Chen ${ }^{4}$, C.C. Liu $^{2}$, S.J. Chou ${ }^{2}$, B.L. Huang', \\ C.I. Kuo ${ }^{2}$, C.K. Wen ${ }^{3}$, L.C. Huang ${ }^{2}$ and W. Fang ${ }^{1}$ \\ ${ }^{1}$ Key Lab for Modern Silvicultural Technology of Zhejiang Province, \\ Zhejiang Forestry University, Zhejiang, China \\ ${ }^{2}$ Institute of Plant and Microbial Biology, Academia Sinica, Taiwan, China \\ ${ }^{3}$ National Key Laboratory of Plant Molecular Genetics, \\ Institute of Plant Physiology and Ecology, \\ Shanghai Institutes for Biological Sciences, \\ Chinese Academy of Sciences, Shanghai, China \\ ${ }^{4}$ Department of Life Sciences, National Cheng Kung University, Taiwan, China \\ ${ }^{5}$ College of Natural Resources and Environment, Beijing Forestry University, \\ Beijing, China \\ Corresponding authors: W. Fang \\ E-mail: zjfufw@163.com
}

Genet. Mol. Res. 9 (2): 1085-1093 (2010)

Received February 21, 2010

Accepted March 20, 2010

Published June 11, 2010

DOI 10.4238/vol9-2gmr804

\begin{abstract}
Unlike other plants, bamboo (Bambusoideae) flowering is an elusive physiological phenomena, because it is unpredictable, longperiodic, gregarious, and uncontrollable; also, bamboo plants usually die after flowering. The flowering mechanism in Arabidopsis thaliana, a eudicot model species, is well established, but it remains unknown in bamboo species. We found 4470 and 3878 expressed sequence tags in the flower bud and vegetative shoot cDNA libraries, respectively, of the bamboo species, Bambusa oldhamii. Different genes were found expressed in bamboo flower buds compared to vegetative shoots, based on the Munich Information Center for Protein Sequences functional categorization; flowering-related genes were also identified in this species. We also identified Arabidopsis flowering-specific homologs
\end{abstract}


that are involved in its photoperiod in this bamboo species, along with autonomous, vernalization and gibberellin-dependent pathways, indicating that bamboos may have a similar mechanism to control floral transition. Some bamboo expressed sequence tags shared high similarity with those of rice, but others did not match any known sequences. Our data lead us to conclude that bamboo may have its own unique flowering genes. This information can help us understand bamboo flowering and provides useful experimental methods to study the mechanisms involved.

Key words: Bamboo; Expressed sequence tag; Flowering; Mechanism

\section{INTRODUCTION}

Bamboos (Bambusoideae) are arborescent and perennial plants, and are divided into approximately 1400 species growing mainly in Asia (Wu and Raven, 2006). Flowering is an important phase in a plant's life cycle. Molecular and genetic analyses in the eudicot model species, Arabidopsis thaliana, revealed that it was a complex network of four pathways that interact to regulate the floral transition (Sablowski, 2007). Flowering of bamboos depends on many factors including environment, nutrition, climate, and their physiological status. Several hypotheses have been proposed for their flowering mechanisms (Janzen, 1976; Gielis et al., 1999; Franklin, 2004). When two genes, DlMADS8 and DIMADS18, cloned from young spikelets of a bamboo species (Dendrocalamus latiflorus) were overexpressed in Arabidopsis, curled leaves and early flowering was exhibited (Tian et al., 2005, 2006). Recently, a cDNA library from the in vitro grown shoots of a bamboo species (Bambusa edulis) was established and 987 ESTs (expressed sequence tags) were identified, but no flowering-related ESTs were specified (Liu et al., 2008).

The EST technique used to identify large numbers of expressed genes rapidly was developed by Adams et al. in 1991. The possible function of similar ESTs in one species could be determined, and more related or novel genes could be identified as compared with ESTs annotated in other species. Based on a recent database from NCBI, bamboos had only 2673 ESTs identified while other species such as Pinus taeda, Populus and A. thaliana, had 328,628, 418,090 and 1,526,133 ESTs, respectively. Therefore, it is necessary to identify more ESTs in bamboos.

In this study, we used the EST technique to determine ESTs in the bamboo species Bambusa oldhamii, and identified 4470 and 3878 ESTs from its flower bud and vegetative shoot cDNA libraries, respectively. All these ESTs were annotated, and some ESTs might act in bamboo flowering when these two cDNA libraries are compared and when compared to those in other species including Arabidopsis and rice. ESTs, which we have identified, provide important information for the study of the bamboo's flowering mechanism.

\section{MATERIAL AND METHODS}

\section{Bamboo tissue culture}

Bamboo tissue cultures were grown from 5-10-mm long shoot tips from newly emerging laterals from healthy, vigorously growing culms of $B$. oldhamii, as described in other study (Huang et al., 1989). Simply, the laterals were dislodged, washed with detergent solution, and 
sterilized by immersion for $10 \mathrm{~min}$ in $0.5 \%(\mathrm{v} / \mathrm{v}) \mathrm{NaOCl}$ solution containing two drops of Tween-20 emulsifier per $100 \mathrm{~mL}$. The sterilized shoots were then rinsed several times with autoclaved distilled water and their tips were excised aseptically under a dissecting microscope. Initiated in 1991, shoot tips in each tube were inoculated in MS medium gelled with Gelrite $(0.25 \%, w / v)$ and supplemented with $1 \mathrm{mg} / \mathrm{L}$ benzyladenine (Sigma, St. Louis, MO, USA) to develop individual plants. The plants were then maintained in the same medium that changed every 28 days. Some of the plants have been flowering since 2001. The flowering buds used in this study were derived from these flowered plants and were subcultured in the medium used for their ancestral plants. The vegetative shoots used in this study were derived from those plants that have never flowered since their initial culture in 1991. All plants were grown under a 16-h light/8-h dark photoperiod indoor at a temperature of $25-27^{\circ} \mathrm{C}$.

\section{RNA isolation and cDNA library construction}

Vegetative shoots and flower buds collected from the cultured plants as described above were ground in liquid nitrogen using a mortar and pestle. Total RNA was isolated with RNAgent (BIOMAN). cDNA libraries were constructed from corresponding vegetative shoots and flower buds using a SMART cDNA Library Construction Kit (BD Biosciences) and cDNA clones were converted to plasmid pDNR-LIB vector according to manufacturer instructions.

\section{EST sequencing, assembly and sequence analysis}

Plasmid DNAs were used as sequence templates. Sequence reaction was performed using the ABI Prism ${ }^{\circledR}$ BigDye $^{\mathrm{TM}}$ Terminator Cycle Sequencing Ready Reaction Kit (version 3.0) with a primer (5'-ATTATACGAAGTTATCAGTCGAC-3') to generate 5'-end sequences of the cDNA clones. Sequencing was done with an ABI PRISM 3700 DNA Sequencer (PE Applied Biosystems).

Sequence data were analyzed by the Phred program (Ewing et al., 1998; Ewing and Green, 1998), and base calls with Phred quality values under 16 were considered to be ambiguous. Sequences were trimmed when five ambiguous bases were observed within 15 continuous bases. The vector-derived sequence was also removed. The clustering of ESTs was performed at the criterion of $95 \%$ identity for 50 bases. ESTs were similarity searched against public protein databases by the BLASTX program (Altschul et al., 1997). Functional categorization of ESTs was performed by aligning translated amino acid sequences with the Munich Information Center for Protein Sequences (MIPS) database (Frishman et al., 2001).

\section{Real-time polymerase chain reaction}

Total RNA of flower buds and vegetative shoots of $B$. oldhamii was extracted with Trizol reagent (Dingguo). First-strand cDNA was synthesized with SuperScript II (Invitrogen) using oligo (dT) ${ }_{20}$ and treated with RNase $\mathrm{H}$. The resulting cDNA was analyzed by real-time polymerase chain reaction (PCR) with an SYBR green kit (Takara) and detected in a Lightcycler according to manufacturer instructions. At least three PCR using the same templates were performed to get average values of expression levels. The PCR conditions were $10 \mathrm{~s}$ at $95^{\circ} \mathrm{C}$, followed by 40 cycles of $5 \mathrm{~s}$ at $95^{\circ} \mathrm{C}$, and $20 \mathrm{~s}$ at $60^{\circ} \mathrm{C}$. The homolog of the Actin gene of B. oldhamii was used as a control. The primer pairs were actin-F (5'-TGGAGACCGCAAAGACGA-3') and actin-R 
(5'-GAAGGATGGCTGGAAGAGGA-3'). The gene specific primer sets were listed as follows: Bo004-F (5'-CGCTTCATGAGGGAGGAAA-3') and Bo004-R (5'-CCTCGTTGCTGTTGGAAG TAGA-3'), Bo009-F (5'-CGCGCTCATCATCTTCTCC-3') and Bo009-R (5'-GTAGTTGCAGGT GCGGTATCTCT-3'), Bo012-F (5'-GCTGAGAAGATGGGGAGGG-3') and Bo012-R (5'-GCTG GAGAAGACGATGAGGG-3'), Bo024-F (5'-CAGCGAACGCAAAGGAAT-3') and Bo024-R (5'-TGATGTGCTTGAAGGACGAA-3'), Bo032-F (5'-CTTGACAATGGGCTGACGAA-3') and Bo032-R (5'-GCGACATCTTGCTGGTGAA-3').

\section{RESULTS}

\section{Bamboo plants growing on culture medium flowered}

Bamboo plants derived from tissue cultures initiated in 1991 and growing on the culture medium, as shown in Figure 1A, began to flower after a 10-year subculture. Inflorescences (spikelets) grew out from their vegetative shoots (Figure 1B). Florets from bamboo plants outdoor and on culture medium possessed all lemma, palea, lodicules, androecium, and gynoecium. The spikelets and florets from plants growing on culture medium were similar to those from outdoor plants but their floral parts were smaller (data not shown). Flower buds derived from bamboo plants growing on culture medium proliferated in the same medium, and vegetative shoots regenerated from the inflorescences (Figure 1C). Flowering of bamboo growing on culture medium provides a useful means to study its flowering mechanism.

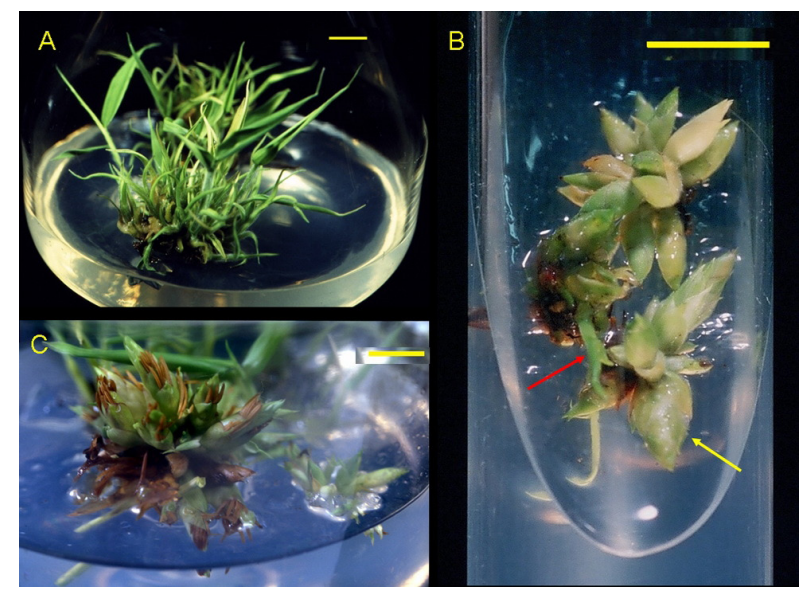

Figure 1. Tissue culture of bamboo (Bambusa oldhamii). A. Vegetative shoot growing on culture medium. B. Bamboo plants flowered in vitro. C. Flower buds proliferated, as indicated with a yellow arrow, and vegetative shoots, as indicated with a red arrow, regenerated from flower buds (scale bar $=1 \mathrm{~cm}$ ).

\section{Different genes present in bamboo flower buds and its vegetative shoots}

To determine the genes responsible for bamboo flowering, we utilized the EST technique to construct cDNA libraries for the flower buds and vegetative shoots of bamboo plants growing on the culture medium. We identified 4470 and 3878 ESTs from flower bud and vegetative shoot 
cDNA libraries, respectively, using DNA sequencing (Table 1). All ESTs from the flower bud cDNA library were grouped into 757 contigs and 2308 singletons that consist of 3065 unigenes, while ESTs from the vegetative shoot cDNA library were grouped into 483 contigs and 1767 singletons that consist of 2250 unigenes. As shown in Table 1, gene redundancy of ESTs in flower bud and vegetative shoot cDNA libraries was 31 and $42 \%$, respectively. Moreover, the percentage of unigenes fell but gene redundant level rose when sequence numbers increased. Compared to the corresponding sequences in rice in the GenBank nr database (TBLASTX, E $\leq 10-5$ ), 47\% ESTs in flower buds and $55 \%$ ESTs in vegetative shoots were found to be similar, respectively.

Table 1. Redundancy of sequences of Bambusa oldhamii flower bud and vegetative shoot cDNA libraries.

\begin{tabular}{|c|c|c|c|c|c|c|c|}
\hline \multirow[b]{2}{*}{ Sequence } & \multicolumn{3}{|c|}{ Flower bud cDNA library } & \multicolumn{4}{|c|}{ Vegetative shoot cDNA library } \\
\hline & Unigene & Percentage & Redundancy & Sequence & Unigene & Percentage & Redundancy \\
\hline 96 & 90 & $94 \%$ & $6 \%$ & 2246 & 1401 & $62 \%$ & $38 \%$ \\
\hline 480 & 394 & $82 \%$ & $18 \%$ & 3878 & 2250 & $58 \%$ & $42 \%$ \\
\hline 1011 & 750 & $74 \%$ & $26 \%$ & & & & \\
\hline 4470 & 3065 & $69 \%$ & $31 \%$ & & & & \\
\hline
\end{tabular}

All ESTs in bamboo flower buds and vegetative shoots were annotated to determine functions of their corresponding unigenes. Putative functions of the bamboo unigenes were classified into 19 categories using the MIPS functional categorization system by aligning them with A. thaliana, as shown in Table 2. Among 19 functional categories, the most abundant category was unknown proteins, representing approximately $25.8 \%$ in flowering buds and $24.0 \%$ in vegetative shoots, respectively. The other proteins were involved in specific functions including metabolism, subcellular localization, transcription, and cellular communication/signal transduction mechanism. The composition of flower bud and vegetative shoot unigene-related proteins was similar, but there were more proteins responsible for cell cycle and DNA processing, protein synthesis, etc., in the flower buds than in the vegetative shoots. Taken together, these data suggest that genes in the flowering buds are different from those in the vegetative shoots in bamboos.

\begin{tabular}{|c|c|c|c|c|}
\hline Function classification & Flower & $\%$ & Shoot & $\%$ \\
\hline 01 Metabolism & 360 & $11.7 \%$ & 297 & $13.2 \%$ \\
\hline 02 Energy & 31 & $1.0 \%$ & 38 & $1.7 \%$ \\
\hline 03 Cell cycle and DNA processing & 105 & $3.4 \%$ & 73 & $3.2 \%$ \\
\hline 04 Transcription & 182 & $5.9 \%$ & 136 & $6.0 \%$ \\
\hline 05 Protein synthesis & 154 & $5.0 \%$ & 111 & $4.9 \%$ \\
\hline 06 Protein fate (folding, modification, destination) & 171 & $5.6 \%$ & 123 & $5.5 \%$ \\
\hline 08 Cellular transport and transport mechanisms & 82 & $2.7 \%$ & 67 & $3.0 \%$ \\
\hline 10 Cellular communication/signal transduction mechanisms & 170 & $5.5 \%$ & 136 & $6.0 \%$ \\
\hline 11 Cell rescue, defense and virulence & 101 & $3.3 \%$ & 86 & $3.8 \%$ \\
\hline 13 Regulation of/interaction with cellular environment & 24 & $0.8 \%$ & 21 & $0.9 \%$ \\
\hline 25 Development (systemic) & 26 & $0.8 \%$ & 24 & $1.1 \%$ \\
\hline 29 Transposable elements, viral and plasmid proteins & 28 & $0.9 \%$ & 18 & $0.8 \%$ \\
\hline 30 Control of cellular organization & 35 & $1.1 \%$ & 29 & $1.3 \%$ \\
\hline 40 Subcellular localization & 229 & $7.5 \%$ & 185 & $8.2 \%$ \\
\hline 63 Protein with binding function or co-factor requirement (structural or catalytic) & 120 & $3.9 \%$ & 85 & $3.8 \%$ \\
\hline 65 Storage protein & 0 & $0.0 \%$ & 1 & $0.0 \%$ \\
\hline 98 Classification not yet clear-cut & 29 & $0.9 \%$ & 33 & $1.5 \%$ \\
\hline 99 Unclassified proteins & 170 & $5.5 \%$ & 106 & $4.7 \%$ \\
\hline The number of "unknown protein" & 792 & $25.8 \%$ & 539 & $24.0 \%$ \\
\hline The number of "No hits found" & 256 & $8.4 \%$ & 142 & $6.3 \%$ \\
\hline Total & 3065 & $100.0 \%$ & 2250 & $100.0 \%$ \\
\hline
\end{tabular}




\section{Unigenes responsible for flowering were observed in bamboo flower buds}

To determine flowering-related genes in bamboos, we analyzed all the unigenes in bamboo flower buds and in their vegetative shoots using TBLASTX. We found "no hits found" for unigenes present in both flower bud and vegetative shoot cDNA libraries, as shown in Table 3. When the factor e is less than 1, 41 "no hits found" unigenes present in the flower buds, while only 33 "no hits found" unigenes were observed in its vegetative shoots. Among the 41 "no hits found" unigenes that are specific for bamboo flower buds, 18 unigenes were specific for bamboos compared to the other plants (data not shown). Thus, it is likely that genes specific for flowering are present in the flower buds, and some genes specific for flowering are present only in bamboos rather than in the other plants.

\begin{tabular}{lccr}
\multicolumn{4}{c}{ Table 3. Comparison of unigenes in Bambusa oldhamii in flower bud and vegetative shoot cDNA libraries. } \\
\hline & $\mathrm{e}<1$ & $\mathrm{e}<0.1$ & $\mathrm{e}<0.001$ \\
\hline Flower specific unigenes & 41 & 805 & 1437 \\
Shoot specific unigenes & 33 & 531 & 968 \\
\hline
\end{tabular}

\section{Flowering-specific homologs of Arabidopsis present in bamboos}

As described earlier, four major signaling pathways of Arabidopsis flowering were suggested (Sablowski, 2007). We compared all ESTs derived from bamboo flowering buds and vegetative shoots with those in Arabidopsis, and found that bamboos had many homologs including CO (CONSTANS), FLD (Flowering locus D), VRN1 (Vernalization1), SPY (Spin$d l y$ ), and PIF3 (Phytochrome interacting factor 3), as shown in Table 4. It is likely that these homologs may be related to bamboo flowering.

\begin{tabular}{|c|c|c|c|c|}
\hline Function of bamboo ESTs & Flower & Shoot & Arabidopsis homolog & Pathway/Function \\
\hline Zinc finger protein & 56 & 51 & CO (CONSTANS) & Long day pathway \\
\hline MADS protein & 16 & 3 & MADS box & Floral promoter \\
\hline RING zinc finger protein & 9 & 2 & $\begin{array}{l}\text { HOS1 (high expression of } \\
\text { osmotically responsive genes } 1 \text { ) }\end{array}$ & Cold signaling \\
\hline MYB transcription factor & 8 & 4 & CCA1 (Circadian Clock Associated 1) & Circadian clock \\
\hline F-box protein (kelch repeat-containing) & 8 & 8 & UFO (unusual floral organ) & Floral promoter \\
\hline WD40-repeat protein & 8 & 10 & FVE & $\begin{array}{l}\text { Autonomous pathway/ } \\
\text { Flower repressor }\end{array}$ \\
\hline Chromatin remodeling factor & 3 & 1 & $\begin{array}{l}\text { PIE } 1 \text { (phytochrome independent } \\
\text { early flowering 1) }\end{array}$ & Floral repressor \\
\hline Histone deacetylase & 3 & 1 & FLD (Flowering locus D) & $\begin{array}{l}\text { Autonomous pathway/ } \\
\text { Flower repressor }\end{array}$ \\
\hline Phytochrome & 3 & 0 & PHY (Phytochrome) & Light perception \\
\hline Casein kinase & 2 & 0 & CK2 (Casein kinase 2) & Circadian clock \\
\hline Gigantea-like protein & 2 & 2 & GI (Gigantea) & Long day pathway \\
\hline Cryptochrome & 2 & 0 & CRY (Cryptochrome) & Light perception \\
\hline Drooping leaf protein & 2 & 0 & DL (Drooping leaf) & Carpel development \\
\hline Polyadenylation factor & 2 & 0 & FY & Autonomous pathway \\
\hline YABBY protein & 2 & 0 & CRC (Crabs claw) & Floral promoter \\
\hline $\mathrm{N}$-acetylglucosaminyl transferase & 1 & 0 & SPY (Spindly) & GA pathway \\
\hline Vernalization independence 4 & 1 & 1 & VIP 4 & Flower repressors \\
\hline AP2 transcription factor & 0 & 1 & TOE $1-2$ (target of eat 1 and 2$)$ & Flower repressors \\
\hline Polycomb group protein & 0 & 1 & VRN1 (Vernalization1) & Vernalization pathway \\
\hline Helix-loop-helix transcription factor & 0 & 1 & PIF3 (Phytochrome interacting factor 3) & Light signaling \\
\hline
\end{tabular}

ESTs = expressed sequence tags; GA pathway = gibberellin-dependent pathway. 


\section{Flowering-related genes significantly expressed in bamboo flower buds}

To examine flowering-related gene expression levels in bamboo flower buds and vegetative shoots, five genes (Bo004, the homolog of CRABS CLAW; Bo009, the homolog of SEPALLATA1; Bo012, the homolog of AGAMOUS; Bo024, the homolog of SEPALLATA3; $B o 032$, the homolog of PISTILLATA) that are randomly selected based on the above sequence analysis were determined by real-time PCR. As shown in Figure 2, their expression levels were all significantly higher in the flower buds than in the vegetative shoots, indicating that these five genes are more active in the flower buds than in the vegetative shoots. These data suggest that these five genes may be involved in bamboo flowering.

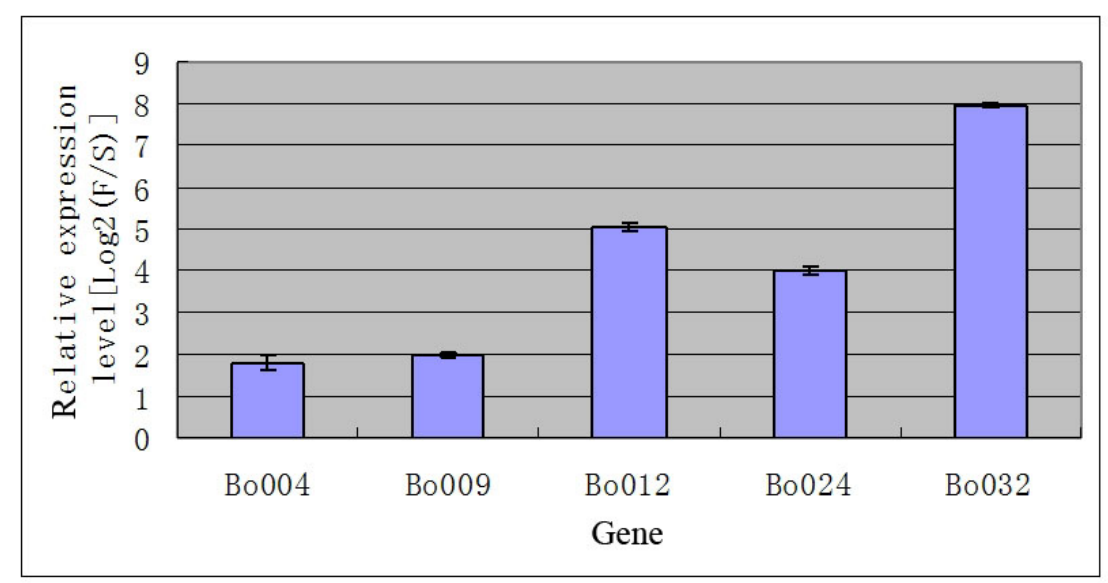

Figure 2. Expression profile of flower specific genes of Bambusa oldhamii by real-time PCR. The relative levels of gene expression are represented in base 2 logarithm. F/S represents the ratio of the gene expression in flower buds versus vegetative shoots.

\section{DISCUSSION}

In vitro bamboo flowering was reported in Bambusa arundinacea, Dendrocalamus brandisii, and $D$. strictus as well as other species but in most of these reports the juvenile shoots that precociously flowered were derived from seedlings (Nadgauda et al., 1990; Ramanayake et al., 2001; Lin et al., 2003). However, we observed in vitro bamboo flowering that occurred in bamboo plants developed from bamboo tissue culture initiated in 1991 and thereafter subcultured continuously on culture medium. It is difficult to study bamboo flowering because in the wild it is unpredictable and factors affecting bamboo flowering are uncontrollable. Also, morphology of spikelets and florets from plants in our experiments was similar to those from outdoor bamboo plants, indicating that bamboo flowering in our culture system is comparable to that in outdoor environments. Furthermore, when the full-length cDNA of Bo009 cloned from B. oldhamii was over expressed in Arabidopsis and rice, the transgenic Arabidopsis and rice exhibited early flowering (data not shown). Taken together, our data thus suggest that our tissue culture system provides a useful means to study the flowering mechanism of bamboo plants. 
We constructed the flower bud and vegetative shoot cDNA libraries of $B$. oldhamii using the EST technique and identified many flower-specific unigenes. Some of those unigenes did not match any known sequences, indicating that bamboo may have its own unique flowering genes (Table 2). Because many identified cDNA clones from bamboo flower buds encoded proteins whose functions are not known, further study is thus required to determine those protein functions. Bamboo ESTs shared high similarity with those of rice, and the relationship between bamboo and rice was closer than that between rice and wheat or barley since the grass family had high colinearity (Moore et al., 1995; Feuillet and Keller, 2002). Therefore, rice genomic information is useful to study flower-related genes or flowering mechanisms in bamboos.

Arabidopsis is a plant whose flowering mechanism is well known and possesses the clearest genetic network of flowering among higher plants. Flowers of grasses and Arabidopsis differ in perianth structure and arrangement. However, grasses have many Arabidopsis flowering-related homologs including $L F Y$ and $S O C 1$, and they also have the corresponding ABCDE model of eudicots with lodicules as modified petals, and palea and lemma as modified sepals, indicating that their flowers probably develop through similar genetic control mechanisms (Ambrose et al., 2000; Tadege et al., 2003; Kater et al., 2006; Alexandre and Hennig, 2008). A flowering model of rice is proposed based on the Arabidopsis flowering model and rice genomic sequences (Goff et al., 2002; Izawa et al., 2003). The flowering models of rice and Arabidopsis present differences, but their frameworks are similar (Izawa et al., 2003; Andersen et al., 2004; Laurie et al., 2004; Winichayakul et al., 2005; Cockram et al., 2007; Izawa, 2007).

Although many bamboo homologs of Arabidopsis flower-related genes were identified by our group and others, bamboo flowering mechanisms remain elusive. To understand molecular mechanisms of bamboo flowering, we identified $L F Y, F T, A P 1, E M F, S O C 1$, and $F C A$ homologs of Aradidopsis from Bamboo (data not shown). The presence of $L F Y, F T$, $A P 1, E M F$, and $F C A$ in B. oldhamii as well as its homologs of Arabidopsis flower-specific genes (Table 4) showed that bamboo had many proteins similar to those that are involved in Arabidopsis flowering mechanisms including photoperiod, autonomous, vernalization, and gibberellin-dependent pathways. Therefore, it is likely that bamboo also have similar mechanisms to control floral transition.

\section{ACKNOWLEDGMENTS}

Research supported by the National Natural Science Foundation of China (grants \#30471416) and the Natural Science Foundation of Zhejiang Province (grants \#Y304345). There are no conflicts of interest in this study. We thank Dr. Xuerong Shi for his critical reading of the manuscript.

\section{REFERENCES}

Adams MD, Kelley JM, Gocayne JD, Dubnick M, et al. (1991). Complementary DNA sequencing: expressed sequence tags and human genome project. Science 252: 1651-1656.

Alexandre CM and Hennig L (2008). FLC or not FLC: the other side of vernalization. J. Exp. Bot. 59: 1127-1135.

Altschul SF, Madden TL, Schaffer AA, Zhang J, et al. (1997). Gapped BLAST and PSI-BLAST: a new generation of protein database search programs. Nucleic Acids Res. 25: 3389-3402. 
Ambrose BA, Lerner DR, Ciceri P, Padilla CM, et al. (2000). Molecular and genetic analyses of the Silkyl gene reveal conservation in floral organ specification between Eudicots and Monocots. Mol. Cell 5: 569-579.

Andersen CH, Jensen CS and Petersen K (2004). Similar genetic switch systems might integrate the floral inductive pathways in dicots and monocots. Trends Plant Sci. 9: 105-107.

Campbell JJN (1985). Bamboo flowering patterns: a global view with special reference to East Asia. J. Am. Bamboo Soc. 6: $17-35$.

Campbell JJN and Qin ZS (1983). Interaction of giant pandas, bamboos and people. J. Am. Bamboo Soc. 4: 1-35.

Cockram J, Jones H, Leigh FJ, O'Sullivan D, et al. (2007). Control of flowering time in temperate cereals: genes, domestication, and sustainable productivity. J. Exp. Bot. 58: 1231-1244.

Ewing B and Green P (1998). Base-calling of automated sequencer traces using Phred. II. Error probabilities. Genome Res. 8: 186-194.

Ewing B, Hillier L, Wendl MC and Green P (1998). Base-calling of automated sequencer traces using Phred. I. Accuracy assessment. Genome Res. 8: 175-185.

Feuillet C and Keller B (2002). Comparative genomics in the grass family: molecular characterization of grass genome structure and evolution. Ann. Bot. 89: 3-10.

Franklin DC (2004). Synchrony and asynchrony: observations and hypotheses for the flowering wave in a long-lived semelparous bamboo. J. Biogeogr. 31: 773-786.

Frishman D, Albermann K, Hani J, Heumann K, et al. (2001). Functional and structural genomics using PEDANT. Bioinformatics 17: 44-57.

Gielis J, Goetghebeur P and Debergh P (1999). Physiological aspects and experimental reversion of flowering in Fargesia murieliae (Poaceae, Bambusoideae). Syst. Geogr. Plants 68: 147-158.

Goff SA, Ricke D, Lan TH, Presting G, et al. (2002). A draft sequence of the rice genome (Oryza sativa L. ssp. japonica). Science 296: 92-100.

Huang LC, Huang BL and Chen WL (1989). Tissue culture investigations of bamboo-IV. Organogenesis leading to adventitious shoots and plants in excised shoot apices. Env. Exp. Bot. 29: 307-315.

Izawa T (2007). Adaptation of flowering-time by natural and artificial selection in Arabidopsis and rice. J Exp. Bot. 58: 3091-3097.

Izawa T, Takahashi Y and Yano M (2003). Comparative biology comes into bloom: genomic and genetic comparison of flowering pathways in rice and Arabidopsis. Curr. Opin. Plant Biol. 6: 113-120.

Janzen DH (1976). Why bamboos wait so long to flower. Annu. Rev. Ecol. Syst. 7: 347-391.

Kater MM, Dreni L and Colombo L (2006). Functional conservation of MADS-box factors controlling floral organ identity in rice and Arabidopsis. J. Exp. Bot. 57: 3433-3444.

Laurie DA, Griffiths S, Dunford RP, Christodoulou V, et al. (2004). Comparative genetic approaches to the identification of flowering time genes in temperate cereals. Field Crop Res. 90: 87-99.

Lin CC, Lin CS and Chang WC (2003). In vitro flowering of Bambusa edulis and subsequent plantlet survival. Plant Cell Tiss. Org. Cult. 72: 71-78.

Liu NT, Wu FH, Tsay HS, Chang WC, et al. (2008). Establishment of a cDNA library from Bambusa edulis Murno in vitro-grown shoots. Plant Cell Tiss. Org. Cult. 95: 21-27.

Moore G, Devos KM, Wang Z and Gale MD (1995). Cereal genome evolution. Grasses, line up and form a circle. Curr. Biol. 5: 737-739.

Nadgauda RS, Parasharami VA and Mascarenhas AF (1990). Precocious flowering and seeding behaviour in tissuecultured bamboos. Nature 344: 335-336.

Ramanayake SMSD, Wanniarachchi WAVR and Tennakoon TMA (2001). Axillary shoot proliferation and in vitro flowering in an adult giant bamboo, Dendrocalamus giganteus Wall Ex Munro. In Vitro Cell Dev. Biol. Plant 337: 661-667.

Sablowski R (2007). Flowering and determinacy in Arabidopsis. J. Exp. Bot. 58: 899-907.

Tadege M, Sheldon CC, Helliwell CA, Upadhyaya NM, et al. (2003). Reciprocal control of flowering time by OsSOC1 in transgenic Arabidopsis and by FLC in transgenic rice. Plant Biotechnol. J. 1:361-369.

Tian B, Chen Y, Yan Y and Li D (2005). Isolation and ectopic expression of a bamboo MADS-box gene. Chin. Sci. Bull. 50: 217-224.

Tian B, Chen Y, Li D and Yan Y (2006). Cloning and characterization of a bamboo Leafy Hull Sterilel homologous gene. DNA Seq. 17: 143-151.

Winichayakul S, Beswick NL, Dean C and Macknight RC (2005). Components of the Arabidopsis autonomous floral promotion pathway, FCA and FY, are conserved in monocots. Funct. Plant Biol. 32: 345-355.

Wu ZY and Raven PH (Editors) (2006). Flora of China: Poaceae. Vol. 22. Science Press and Missouri Botanical Garden, Beijing. 